\title{
In silico Docking Studies and Potential Lead Identification against JNK3 for Alzheimer's Disease
}

\author{
Nishtha Singh, Sonal Upadhyay, Ankur Jaiswar, Nidhi Mishra* \\ Department of Applied Science, Indian Institute of Information Technology, Allahabad, Deoghat, Jhalwa, Uttar Pradesh, INDIA.
}

\begin{abstract}
Background: Alzheimer's Disease (AD) is a neuron related brain disorder leading to reasoning and memory loss. There is no specific cure identified for AD. JNK3 (c-Jun N-terminal kinase /stress-activated protein kinase) are highly revealed within the central nervous system, particularly neurons, playing vital role in functioning of brain. JNK3 hyper phosphorylation is a very common conclusion in neurodegenerative diseases. JNK3 in turn hyper phosphorylates Amyloid Precursor Protein (APP) which leads to the formation of Amyloid $\beta$ peptides (an inductive agent of Alzheimer's disease). Methods: Protein JNK-3 (PDB ID: 3KVX) was retrieved from protein data bank and later we docked a library of compounds against it. These were further validated by ADMET studies. Results: Thus, docking inhibitors of JNK3 may provide a promising sanitive approach. Based on best docking
\end{abstract}

score and glide score a potential lead is identified against JNK3. Conclusion: Inhibiting JNK-3 may lead to less production of amyloid $\beta$ peptides, thus reducing the risk of Alzheimer's disease.

Key words: JNK3, Alzheimer's disease, Amyloid $\beta$ peptides.

Correspondence

Nidhi Mishra,

Department of Applied Science, Indian Institute of Information Technology, Allahabad, Deoghat, Jhalwa-211012, Uttar Pradesh, INDIA.

Phone no: +910532 2922540

Email: nidhimishra@iiita.ac.in

DOI: 10.5330/ijpi.2019.4.41

\section{INTRODUCTION}

Alzheimer's disease (a form of 'dementia') a neurodegenerative disease leading to degeneration and loss of neurons is affecting the people across the globe. ${ }^{1}$ According to World Alzheimer Report 2015 around 46.8 million people are suffering from dementia worldwide and it is reported that this figure will double every 20 years. Although, at present it is affecting America (9.4 million), Europe (10.5 million), Africa (4.0 million) and Asia (22.9 million). ${ }^{2}$ The first case of Alzheimer was identified by Alois Alzheimer in 1901 in a 50 year old woman. ${ }^{3}$ The rate of progression of this disease varies. The process of developing a drug usually takes a duration of 10-15 years starting from lead identification to clinical tests and the approvement by Food and Drug Administration (FDA). Approximately 1 billion USD is the average expense on one new drug. ${ }^{4}$ Thus, potential lead against Alzheimer's disease can be identified using Computer Aided Drug Design (CADD) and this study can be advanced further in vitro and in vivo studies as a potent anti-alzheimer drug. Alzheimer's disease is found to be increasing in rapidly aging population (>60 years). And the treatment for Alzheimer disease remains elusive.

Amyloid $\beta$ peptides $(\mathrm{A} \beta 42)$ have been found to play a vital role in $\mathrm{Al}$ zheimer's disease $(\mathrm{AD})$. In normal conditions these peptides are produced in minute amounts and removed from the body but under pathogenic conditions these accumulate in form of oligomers. ${ }^{5}$ These peptides activate AMPK pathway, leading to the obstruction of them TOR pathway. ${ }^{6}$ This inhibition promotes widespread stress in ER, leading to activation of Unfolded Protein Response (UPR) and this ER stress as a part of UPR activates JNK3. And then APP is phosphorylated at site T668 by JNK3 and thereby from cell surface, APP internalize within endosome. On outer surface of endosome an enzyme $\beta$ secretes is present which produces Amyloid $\beta$ peptides and increases its processing which results in intensified yield of Amyloid $\boldsymbol{\beta}$ peptides, whose accretion leads to Alzheimer's disease. Therefore, targeting JNK3 may decrease the produc- tion of Amyloid $\boldsymbol{\beta}$ peptides. ${ }^{7}$ Thus, overall plaque loads and A $\beta 42$ levels are reduced by deletion of JNK3. ${ }^{8}$

\section{METHODS}

\section{Preparation of protein structure}

The X- Ray structure of target protein JNK 3 at $2.40 \AA$ resolution (PDB ID: $3 \mathrm{KVX})$ is a Transferees obtained from RCSB Protein Data Bank (RCSB PDB). ${ }^{9}$ The protein is processed by 'Protein Preparation Wizard' of Maestro, version 9.7, Schrödinger LLC, New York, 2014. ${ }^{10}$ Prior to beginning the processing of protein, removal of heteroatoms and water molecules is done from the protein crystal structure 3KVX. Modifications are made which includes stabilization of charges, removal of the water molecules, filling in the missing residues, according to the parameters that are available. H-bonds network are optimized by implementing the $\mathrm{H}$-bond assignment tool. The energy is minimized by utilizing RMSD: $3 \AA$ and force field: OPLS_2005. After the modification is made the receptor (target) should be biologically active and stable.

\section{Active Site Prediction}

The active binding site of target receptor $3 \mathrm{KVX}$ is recognized by utilizing 'Sitemap', Schrödinger LLC, New York, 2014. ${ }^{11}$ To differentiate between known binding sites Site score is used which is an important property produced by Sitemap. For Receptor Grid Generation in JNK 3 receptor active binding site with best site score is taken as an imperative.

\section{Grid preparation}

'Receptor Grid Generation' tool of Glide manual Schrödinger LLC, New York, 2014 employed to produce grid which ascertains the structure of receptor by cutting out any ligand that may be co-crystallized, fix on location and range of active site. ${ }^{12}$ Grid point's level for $\mathrm{X}, \mathrm{Y}, \mathrm{Z}$ axis 
$(-23,30,10)$ for $3 \mathrm{KVX}$. And the grid is generated using the force field OPLS_2005.

\section{Similar Compound Library Selection and Preparation}

JNK3 inhibitor JNK-IN-7 and JNK-IN-7 extended library of compounds were downloaded in SDF format. The geometry of ligands is minimized by using the force field OPLS_2005. QikProp, Schrödinger, LLC, New York, 2014 was adopted for virtual screening of the compounds (obtained from database) throughout their preparation and moreover to evaluate the properties of ADMET like absorption, distribution, metabolism and excretion and toxicity. ${ }^{13}$ Ligand's Pharmaokinetic profile is insured by one of the factors: Lipinsky's rule of five.

\section{Virtual screening}

Virtual screening is a convenient procedure to identify and position potential inhibitors against the target protein according to their ranks from a database of diverse compounds. Taking into consideration the active binding site of $3 \mathrm{KVX}$ virtual screening was accomplished by employing the compound database. The compounds were docked using Extra Precision (XP) phase. Glide module of XP visualize evaluates several particular interactions like Hydrogen bonds, Protein - ligand interactions, Internal energy, RMSD (root mean square deviation ) and hydrophobic interactions. $^{14}$

\section{ADMET Screening}

The ADMET (Absorption, Distribution, Metabolism, Excretion and Toxicity) property of screened inhibitors was estimated by utilizing QikProp program. The program further estimates pharmaceutical properties and physical descriptors. It assesses the lead compound which is imperative for rational drug design on the base of Lipinski's Rule of Five. ${ }^{13}$

\section{RESULTS}

Altogether, 1935 molecules were screened against the target protein. At the beginning compounds with reactive functional groups and Molecular weight greater than 500 were refined. The eventual molecules after filtering were docked into the active site of $3 \mathrm{KVX}$. The features of screened lead compounds have been summed up in Table 1 and the 2-D structure of top compound and reference molecule is shown in Table 2. Glide emodel was used to grade the ligands poses and Glide score was utilized as scoring function. Alike docking specifications for JNK-IN-7 $(57340685)^{15}$ are also delineated in Table 1 for comparison.

Physical descriptors and pharmaceutical properties of lead compound was explored using QikProp tool as demonstrated in Table 3. The parameters depicted in table are important descriptors. The top ranked screened lead compound was in tolerant range of Lipinsky's Rule of five, depicting their possibility for usage as medicine like drugs. This study can be advanced further using in vitro and in vivo studies as a potent anti-alzheimer drug.

\section{DISCUSSION}

Combined in silico approaches were applied to screen chemical entities against Alzheimer's disease causing protein. Docking study was employed to evaluate the binding interactions of these compounds with the receptor.

The docking studies specify that the topmost screened compounds exhibited strong H-bonding with the target protein. Based on Docking Score, Glide g Score and Glide emodel compound ID labeled 19689560 is more effective against JNK3 receptor (3KVX) as compared to reported inhibitor JNK-IN-7 (57340685).
Table 1: Binding efficiency comparison based on Docking Score, Glide g Score and Glide emodel.

\begin{tabular}{cccc}
\hline ID NO. & Docking Score & Glide g Score & Glide emodel \\
\hline 19689590 & -12.711 & -12.726 & -87.145 \\
38390094 & -10.264 & -10.264 & -83.910 \\
25138031 & -10.138 & -10.151 & -92.497 \\
32648029 & -10.056 & -10.056 & -85.548 \\
87057812 & -9.883 & -9.883 & -74.821 \\
57340685 & -10.41 & -10.305 & -88.656 \\
\hline
\end{tabular}

Table 2: 2-D structure and 2-D interaction diagram of top ranked screened compound and reference molecule respectively.

ID NO.
19689590
57340685

Amino acid residue of screened compound MET 149 forms H-bond (backbone) with the ligand. Hydrophobic bonds are formed with ILE 70, ALA 91, ILE 124, LEU 206, ALA 74, MET 220 amino acid residues. Polar interactions are also observed with residues like ASN 194, SER 193 and SER 72.

Compound ID 70973132, is the best docked molecule according to docking energy and glide score. In the ADMET studies, the percentage oral absorption of this compound comes out to be $100 \%$, which is unacceptable. The partition coefficient of top ranked compound id 70973132 is the highest, predicting its lipid solubility and better permeation across cell membrane. QPlog KP predicted skin permeability for which values are in negative for the top ranked molecules which rules out skin permeability.

The top ranked molecule forms Hydrogen bonds with the help of $\mathrm{OH}$ and NH groups with Asp, Gly, Meth etc. The presence of polar interactions and hydrophobic bonds results in ligand binding to the receptor. The number of hydrogen bonds are responsible for binding of ligand with the receptor site. According to ADMET studies and docking energy 
Table 3: ADMET properties of screened compounds.

\begin{tabular}{|c|c|c|c|c|c|c|}
\hline $\begin{array}{l}\text { ID } \\
\text { NO }\end{array}$ & $\begin{array}{l}\text { QP } \\
\log S\end{array}$ & $\begin{array}{c}\text { QP } \\
\text { log- HERG }\end{array}$ & QPP CaCo & $\begin{array}{c}Q P \\
\log B B\end{array}$ & QPPM-DCK & $\begin{array}{c}\% \text { Human Oral } \\
\text { Absorption }\end{array}$ \\
\hline 19689590 & -4.996 & -7.200 & 59.313 & -3.027 & 23.349 & 70.789 \\
\hline 38390094 & -5.582 & -6.883 & 632.499 & -0.905 & 545.62 & 96.700 \\
\hline 25138031 & -4.923 & -6.689 & 92.778 & -2.129 & 37.869 & 73.040 \\
\hline 32648029 & -4.699 & -6.627 & 615.540 & -0.931 & 292.79 & 91.715 \\
\hline 87057812 & -7.182 & -7.366 & 1141.3 & -0.389 & 5941.9 & 100.00 \\
\hline
\end{tabular}

- Aqueous solubility, Q $\log$ S. Should be in the range from -6.5 to 0.5 Predicted IC $_{50}$ value for blockage of HERG K+ channels (.>-5).

- Predicted apparent Caco-2 cell permeability in nm/sec. Caco-2 cells are the model for the gut blood barrier (25-500).

- $\quad$ Predicted brain/blood partition coefficient. (-3.0 - 1.2).

- $\quad$ Predicted apparent MDCK cell permeability in $\mathrm{nm} / \mathrm{sec}$. (<25-poor and $>500$-great)

- Human oral absorption percentage in GI $( \pm 20 \%)$, should not be less than 25 .

both, the compound ID 19967030 and67214420 are predicted to be the best docked drug candidate. We have obtained good results in comparison to reported lead compound JNK-IN-7 during this computational study. This is an indication that these compounds can be developed as drug candidates in future although further studies need to be done.

\section{CONCLUSION}

In conclusion, the outcome of the study distinctly depicted that screened compound i.e. of ID no. 19689590 is better inhibitor in comparison to reported lead JNK-IN-7 for JNK3 protein targets. This conclusion revealed that JNK inhibitor JNK-IN-7 and screened lead compound have the ability to bind to JNK3 and inhibit its hyper phosphorylation.

\section{ACKNOWLEDGEMENT}

The authors acknowledge Department of Applied Sciences, IIIT-Allahabad for providing necessary computational facilities.

\section{CONFLICT OF INTEREST}

The authors declare no conflict of interest.

\section{REFERENCES}

1. Lansdall CJ. An effective treatment for Alzheimer's disease must consider both amyloid and tau. Bioscience Horizons: Int J Stud Res. 2014:1;7.

2. Alzheimer's Disease International. The global impact of dementia. 2019. http:// www.alz.co.uk/sites/default/files/pdfs/global-impact-dementia-infographic.pdf/
Hippius H, Neundörfer G. The discovery of Alzheimer's disease. Dialogues Clin Neurosci. 2003:5(1):101.

3. Viet MH. Numerical study of protein-ligand binding: Implications for alzheimer's disease and influenza virus. (Doctoral dissertation, Ph. D. thesis). 2012;1-131.

4. Storey E, Cappai R. The amyloid precursor protein of Alzheimer's disease and the Abeta peptide. Neuropathol Appl Neurobio. 1999;25(2):81-97.

5. Nixon RA, Yang DS. Autophagy failure in Alzheimer's disease-locating the primary defect. Neurobiol Dis. 2011;43(1):38-45

6. Yoon SO, Park DJ, Ryu JC, Ozer HG, Tep C, Shin YJ, et al. JNK3 perpetuates metabolic stress induced by A $\beta$ peptides. Neuron. 2012;75(5):824-37.

7. Antoniou X, Falconi M, DiMarino D, Borsello T. JNK3 as a therapeutic target for neurodegenerative diseases. J Alzheimer's Dis. 2011;24(4):633-42.

8. Berman HM, Bourne PE, Westbrook J, Zardecki C. The protein data bank. In Prot St. 2003;18:394-410. CRC Press.

9. Sastry GM, Adzhigirey M, Day T, Annabhimoju R, Sherman W. Protein and ligand preparation: parameters, protocols and influence on virtual screening enrichments. J of Computer-aided Mol Des. 2013;27(3):221-34

10. Halgren TA. Identifying and characterizing binding sites and assessing druggability. J Chem Inf Model. 2009;49(2):377-89.

11. Halgren TA, Murphy RB, Friesner RA, Beard HS, Frye LL, PollardWT, et al. Glide: A new approach for rapid, accurate docking and scoring. 2. Enrichment factors in database screening. J Med Chem. 2004;47(7):1750-9.

12. Lindorff-Larsen K, Piana S, Palmo K, Maragakis $P$, Klepeis JL, Dror RO, et al. Improved side-chain torsion potentials for the Amber ff99SB protein force field. Proteins: Str, Func and Bioinf. 2010;78(8):1950-8.

13. Raj U, Varadwaj PK. Flavonoids as multi-target inhibitors for proteins associated with Ebola virus: In silico discovery using virtual screening and molecular docking studies. Interdisci Sci: Comput Life Sciences. 2016;8(2):132-41.

14. Zhang T, Inesta-Vaquera F, Niepel M, Zhang J, Ficarro SB, Machleidt T, Xie T, et al. Discovery of potent and selective covalent inhibitors of JNK. Chem and Biol. $2012 ; 19(1): 140-54$ 